\title{
Après la lutte
}

Les itinéraires de Pierre Poujade et de Gérard Nicoud ou l'instrumentalisation différenciée du label protestataire

After the struggle: the careers of Pierre Poujade and Gérard Nicoud, or the differentiated instrumentalization of protest label

\section{Marc Milet}

\section{(2) OpenEdition}

Journals

\section{Édition électronique}

URL : http://journals.openedition.org/conflits/18129

DOI : $10.4000 /$ conflits. 18129

ISSN : $1777-5345$

\section{Éditeur :}

CCLS - Centre d'études sur les conflits lilberté et sécurité, L'Harmattan

\section{Édition imprimée}

Date de publication : 5 septembre 2011

Pagination : 151-171

ISBN : 978-2-296-56086-4

ISSN : 1157-996X

\section{Référence électronique}

Marc Milet, «Après la lutte », Cultures \& Conflits [En ligne], 81-82 | Printemps/Été 2011, mis en ligne le 05 septembre 2012, consulté le 30 mars 2021. URL : http://journals.openedition.org/conflits/18129 ; DOI : https://doi.org/10.4000/conflits.18129 


\section{Après la lutte}

\section{Les itinéraires de Pierre Poujade et de Gérard Nicoud ou l'instrumentalisation différenciée du label protestataire}

\section{Marc MILET 1}

Marc Milet est maître de conférences en science politique à l'Université Panthéon Assas, rattaché au CERSA. Il a notamment publié divers travaux relatifs à l'action collective du petit patronat et travaille actuellement sur l'européanisation des groupes de défense des TPE et PME.

Dierre Poujade et Gérard Nicoud incarnent les deux grandes vagues de en France à quinze ans d'intervalle, et issues respectivement d'un mouvement d'opposition aux contrôles fiscaux et de la contestation des effets de la mise en œuvre du régime d'assurance maladie obligatoire des travailleurs non salariés 2. Les deux grands cycles de mobilisation des commerçants et artisans (autour des années 1953-1962 pour le premier et 1969-1977 pour le second) ont donné lieu à de riches études qui offrent à ce jour une connaissance relativement précise des conditions de structuration de l'action collective ${ }^{3}$, de l'importance des interactions avec les agents de l'État sur la radicalisation de ces mouvements, et de leurs implications sur l'action publique 4 .

Pour autant, la qualification générique communément retenue, celle de mouvements de type "protestataire », si elle précise la dimension conflictuelle et d'opposition au pouvoir et aux politiques publiques portée par ces mobili-

1. Je remercie Lorenzo Barrault, Caroline Frau, et les lecteurs anonymes pour leurs remarques sur une version antérieure de ce texte.

2. Pour une approche comparée, cf. Gresle F., "Les petits patrons et la tentation activiste ", in Lavau G., Grunberg G., Mayer N. (ed.), L'univers politique des classes moyennes, Paris, PFNSP, 1983, pp. 293-312.

3. Cf. Hoffman S. et alii., Le mouvement Poujade, Paris, Armand Colin, 1965 ; Gresle F., Indépendants et petits patrons. Pérennité et transformations d'une classe sociale, Thèse d'État en sociologie, 2 vol., Paris-Lille, 1980 ; Vinen R., " Faire pression sur les groupes de pression », in Garrigues J. (ed.), Les Groupes de pression dans la vie politique contemporaine en France et aux Etats-Unis de 1820 à nos jours, Rennes, PUR, 2002, pp. 155-165.

4. Notamment voir Souillac R., Le monvement Poujade. De la défense professionnelle au populisme nationaliste (1953-1962), Paris, Presses de Sciences Po, 2007. 
sations 5, n'en conduit pas moins à minimiser la radicalité des formes d'action employées (tels le recours au plasticage et à la séquestration, la dérive en manifestations violentes). Afin de contribuer à combler ce manque, nous proposons pour l'heure d'interroger ce rapport à la violence au prisme de la trajectoire sociale et professionnelle des deux leaders protestataires.

Le recours à une analyse processuelle 6 fondée sur des récits de vie 7 , reconstruits de manière analytique à partir des propres écrits de Pierre Poujade et de la consultation de l'organe de publication de son organisation, recueillis par entretien narratif 8 pour Gérard Nicoud, permet de mettre à jour les carrières ${ }^{9}$ militantes et professionnelles des deux leaders et offre l'occasion de resituer le moment de lutte dans le continuum de leurs parcours individuels, familiaux et professionnels, et de montrer dans quelle mesure leurs itinéraires demeurent largement tributaires des conditions spécifiques de ce moment violent.

La présentation du parcours postérieur au moment de lutte permet en l'espèce d'évaluer le rapport au passé « activiste » qui fonde en définitive un référent à la fois subi, car perpétuellement rappelé aux acteurs, mais dont la teneur même conduit à une instrumentalisation différenciée du capital militant acquis par la lutte. En effet, Pierre Poujade, absent pour l'essentiel des principales actions radicales menées par son mouvement, s'apparente à un agent modérateur d'une violence qu'il tend à maintenir pour sa part dans l'ordre du discours ${ }^{10}$. Gérard Nicoud quant à lui n’hésite pas à participer directement à certains coups de force et personnalise dès lors la défense de la cause par la violence.

Au-delà d'indéniables convergences de parcours, dont la tentative d'investissement réitérée de l'arène électorale n'est pas la moindre, et à laquelle s'ajoute la (re)construction commune chez les deux hommes d'une cohérence biographique établie dans la défense supposée immuable des «petits », cette différenciation va dès lors peser durablement dans les disponibilités d'une réorientation personnelle et professionnelle. Tandis que Pierre Poujade prolonge le rapport ambivalent avec l'État dans l'élaboration d'un véritable système militant néo-patrimonialiste ${ }^{11}$ fondé sur l'appui étatique et politique, Gérard Nicoud réutilise pour sa part le référent symbolique violent à travers

5. Voir Siméant J., notice "protestation », Lexique de science politique, Paris, Dalloz 2008, p. 433. Sur la catégorie « protestataire » des formes d'action, cf. Mayer N., Sociologie des comportements politiques, Paris, Armand Colin, 2010, pp. 218-226.

6. Fillieule O., "Propositions pour une analyse processuelle de l'engagement individuel », Revue française de science politique, vol. 51, $\mathrm{n}^{\circ}$ 1, 2001, pp. 199-215.

7. Bertaux D., Le récit de vie, Paris, Armand Colin, 2005.

8. Entretien enregistré, 24 janvier 2008, un restaurant de Neuilly sur Seine (2h30).

9. Hugues E., Le regard sociologique. Essais choisis, Paris, éd. de l'EHESS, 1996.

10. Il choisit ainsi, face aux actions en justice intentées contre lui, de minimiser ses appels à la désobéissance civile, et d'indiquer : "nous avons toujours répudié la violence ", Fraternité française (désormais $F F$ ), 8 oct. 1955, p. 2. 
la création d'un rôle inédit du syndicalisme petit patronal : le médiateur fiscal auprès de l'administration.

\section{Désescalade de la violence et reconversions militantes}

Le poujadisme corporatiste se définit dés l'été 1953 par des actions collectives d'opposition aux contrôles fiscaux et aux ventes sur saisie, poursuivies par la grève de l'impôt (au premier semestre 1955), avant que n'intervienne plus tardivement une véritable "situation insurrectionnelle » à l'été et à l'automne 1955. Le mouvement de la Tour du Pin naît pour sa part directement d'un coup de force : celui de la prise de dossiers fiscaux à la perception de la Tour du Pin, qui fait connaittre nationalement le mouvement le 9 avril 196912. Ce mouvement, bientôt structuré en CID-UNATI (Comité d'information et de défense - Union nationale des artisans et travailleurs indépendants) se définit en cela dès l'origine dans et par la violence alors même que celle-ci n'est qu'incidente au poujadisme et que s'y substitue dès lors, dans la mémoire collective même, un référent " protestataire " d'un mouvement en « négatif » 13 . Cet élément de différenciation induit, comme on le verra, des implications fondamentales sur la reconversion des deux leaders.

\section{Les conditions de sortie de la violence}

Les conditions de sortie de la violence s'effectuent selon deux voies distinctes.

Effets de trajectoires : investissement de l'arène électorale versus institutionnalisation du mouvement. La mobilisation poujadiste anti-fiscale se solde par un résultat en demi teinte : la reconnaissance statutaire qui fut au cœur de la fronde favorise le désengagement militant d'autant que les ajustements de la loi fiscale de novembre 1954 sont relativement mesurés et que l'amnistie n'est pas obtenue. L'investissement de la compétition électorale par l'UDCA (l’Union de Défense des Commerçants et Artisans), décidé en novembre 1955, n'est pas la conséquence inéluctable et «naturelle » du processus de politisation entamé quelques mois plus tôt, mais résulte bien directement de l'essoufflement des autres formes de mobilisation ${ }^{14}$. Le répertoire d'action est immanquablement conditionné par l'investissement de l'arène électorale qui contribue aussi à élever le niveau d'enjeu pour le groupement en cas d'inter-

11. Les diverses ressources militantes, conjuguées à l'appui des services de l'État servent le développement d'une activité économique et d'un patrimoine personnel qui offre en retour une visibilité de l'action collective.

12. Le coup d'éclat intervient après la tenue de multiples réunions publiques, durant l'hiver 19681969, Après le coup d'éclat s'ensuivent des affrontements violents avec les forces de l'ordre à Bourgain-Jallieu, prolongés quelques mois plus tard par "l'enlèvement » des agents des Renseignements généraux lors d'un meeting le 25 septembre 1969.

13. Sur les usages du terme, voir Collovald A. "Histoire d'un mot de passe : le poujadisme. Contribution à une analyse des "ismes" ", Genèses, 1991, n 3, notamment p. 98.

14. Voir en ce sens Souillac R., op. cit., p. 176. 
diction. Mais pour autant, le changement d'arène ne signifie aucunement l'arrêt des violences.

En premier lieu, s'opère une réorientation, partielle, de la cible et des formes d'action qui passent des agents de l'administration aux concurrents politiques ${ }^{15}$, et des obstructions aux contrôles fiscaux aux meetings de campagnes électorales 16. Mais une fois passé le succès électoral de 1956, l'essoufflement du mouvement (marqué par l'échec de Poujade lui-même à la députation en 1957) correspond bien au reflux d'un recours global à la violence qui s'accompagne alors d'un processus de radicalisation de la seule "minorité activiste ${ }^{17}$, perceptible dans le durcissement du discours poujadiste anti-système et la routinisation des formes d'action utilisées (attentats et micro-mouvements insurrectionnels se succèdent en 1957 et 1958). Après le changement de régime politique, le « repli groupusculaire » de l'UDCA renvoie à une dernière phase de violence, désormais uniquement circonscrite aux membres (ex)poujadistes de l'OAS (l'organisation de l'armée secrète) en rupture avec l'organisation ${ }^{18}$. L'indéniable succès du poujadisme au sein des Chambres consulaires (de commerce et des métiers) ${ }^{19}$ se révèle éphémère. L'emprise du mouvement au sein des Chambres des métiers est freinée par le principe de renouvellement partiel des élus qui tend à amoindrir sa percée, puis par une modification du régime électoral intervenue en 1959.

La désescalade du recours massif à la violence par le CID-UNATI s'inscrit dans un processus radicalement différent. Il tient tout d'abord aux multiples succès électoraux du mouvement, lors des élections des Chambres de commerce en décembre 1970, des Chambres de métiers de novembre 1971, et des Caisses d'assurance maladie en décembre 1972. Les bons résultats rendent possible une institutionnalisation du groupe protestataire en quête de voie de sortie d'une action directe qui ne peut mobiliser durablement. Les représentants du mouvement se refusent également à toute politisation en se référant à l'échec du poujadisme électoral. Dans le même temps, le pouvoir politique est soucieux de disposer d'un interlocuteur présentable afin de sortir de la crise, comme en témoigne l'épisode du report de l'incarcération de Nicoud afin d'éviter de priver l'État d'un interlocuteur officiel. Après la sortie de prison du leader du CID-UNATI (en juillet 1972, du fait de la grâce présidentielle) peut s'engager la voie de la normalisation. Le congrès du Mans, en janvier 1973, entérine le changement de style d'un leader qui incline à la « responsabi-

15. Voir sur les actions contre les ministres, MM. Faure et Mitterrand, Poujade P., L'histoire sans masque, Cestas, Élytis éd., 2003, pp. 90-98.

16. Souillac R., op. cit., p. 93.

17. Sur ce processus bien connu de la radicalisation minoritaire dans une phase de désescalade de la violence, voir dans un contexte différent Della Porta D., Social movements, political violence and the state. A comparative analysis of Italy and Germany, Cambridge, Cambridge University Press, 2004 (1ère éd. 1995).

18. Sur ces processus voir Souillac R., op. cit., pp. 276 et suiv. ; pp. 357 et suiv. ; pp. 371 et suiv.

19. Voir Magliulo B., Les chambres de métiers, Paris, PUF, 1985 ; Zarca B., L'artisanat français : du métier traditionnel au groupe social, Paris, Economica, 1986, pp. 89-91. 
lité, la compétence et l'honorabilité » 20 . Sa rencontre avec le ministre de tutelle dès l'été 1972 manifeste sa reconnaissance en tant qu'interlocuteur légitime. Ce statut se trouve consolidé par le succès aux élections aux Caisses régionales d'assurance maladie (avec $52 \%$ des sièges), ce qui permet à Gérard Nicoud d'accéder à la présidence de la CANAM (Caisse Nationale d'Assurance Maladie des travailleurs non salariés) en juillet 1974. Désormais, le mouvement se démarque des mots d'ordre violents dans le cadre d'un partenariat qui mène notamment à l'adoption de la législation Royer ainsi qu'au vote de l'amnistie pour les refus de paiement de cotisations. Après la décision du président de la République nouvellement élu, Valéry Giscard d'Estaing, de recevoir le leader d'une organisation devenue représentative, le titre choisi par l'Objectif, le magazine du CID-UNATI, « de la prison à l'Elysée » ${ }^{21}$ rappelle explicitement le chemin parcouru. L'organisation a prolongé la mutation engagée au Mans lors du VII congrès d'octobre 1975, marqué par «l'examen de conscience » et l'entérinement du changement. Cette mutation s'affirme très nettement dès la fin 1973 dans la teneur même des rubriques consacrées par l'organe de l'organisation, l'Objectif, à la « vie départementale » du mouvement : le minutieux panorama des « faits d'armes » (procès intentés, actions violentes menées) qui y était jusqu’alors présenté se mue en un compte-rendu de la vie de l'organisation, ponctuée désormais par la litanie des réunions et fêtes militantes locales 22 .

L'effet de normalisation des comportements induit par cette nouvelle position occupée par le mouvement et son leader demeure toutefois relatif. Car cet accès aux instances consultatives et à la représentativité ne définit pas tant la mise en ouvre d'une «contestation constructive », telle qu'énoncée dans les discours ${ }^{23}$, que l'édification d'un rôle consulaire inédit et bybride car assis sur une combinaison, tout à fait originale, de ressources internes et externes à l'institution. Ainsi s'établissent vers 1974-1976 des formes d'action renouvelées, telles que l'occupation des Chambres de commerce et de métiers par des militants extérieurs du CID-UNATI, en accord et avec la complicité de ses représentants élus, ainsi que l'exercice de pratiques déviantes aux règles $\mathrm{du}$ jeu consulaire ordinaire (prises de parole intempestives, pression internes et externes pour contraindre des présidents « mal élus » à la démission). Le mouvement cherche ainsi à combiner un répertoire d'action conventionnel, établi dans le cadre des relations de négociation et de rencontres institutionnelles, avec le maintien d'un recours aux formes d'actions radicales et illégales originaires. Ce double positionnement s'avère cependant difficilement tenable et explique pour une grande part la démission de Gérard Nicoud de la présidence de la CANAM moins d'un an après son élection.

20. Gresle F., Indépendants et petits patrons, op. cit.

21. L'Objectif, $\mathrm{n}^{\circ}$ 56, nov-déc. 1975.

22. Evolution particulièrement notable dans les numéros de 1974.

23. Le slogan, à la fin des années 60 , est commun au CID-UNATI et à l'UDCA. 
L'effet d'institutionnalisation n'est cependant pas non plus demeuré étranger aux représentants poujadistes élus au sein des Chambres consulaires au milieu des années 1950. Il s'est traduit cependant le plus souvent par un processus de ralliement (ou de retour) en cours de mandat des élus aux organisations syndicales traditionnelles. La normalisation du mouvement n'a, a contrario, aucunement joué sur le processus de sortie de la violence de l'UDCA, car l'abandon de la posture protestataire pour l'édification d'une relation de type néo-corporatiste avec l'État 24 est très postérieure, comme on le verra, à la fois au «moment poujadiste » et à celui du déclin, déjà largement entamé, de l'organisation.

\section{Rapports différenciés du mouvement et de son leader avec la désescalade.} Le déclin du recours ordinaire à la violence suit le déclin du mouvement poujadiste. Alors qu'il s'est rallié au régime de la ve République au milieu des années soixante (infra) et que bientôt l'attention nicoudiste va contribuer à afficher ce recentrage, Pierre Poujade relit son engagement à la lumière de la réorientation de sa carrière militante. Il propose une interprétation historiquement située du recours à l'action violente (« n'oubliez pas que pour avoir voulu faire la guerre de 1939 avec les conceptions des héros de 1914, la France l'a perdue » écrit-il ${ }^{25}$ ) qui le mène aussi à établir à la fois une surévaluation de la dynamique stratégique du recours passé à la violence (par la référence à de "nouvelles méthodes d'action ») ainsi qu'à réécrire la nature du combat poujadiste en choisissant des citations ciblées, telle celle relative à « l'attachement fondamental aux principes » 26 , prononcées au plus fort de la lutte. Le nouveau rapport au régime et à la violence transforme la rhétorique poujadiste et la nature de la cible des attaques verbales : les révoltés de l'Isère sont ainsi apparentés à un « gang nouveau du Dauphiné » qui pratique le « gangstérisme 27 ». De même, Poujade concède encore des années plus tard l'effet d'âge ${ }^{28}$ sur son changement de mode d'action : "Si j'avais 30 ans, avant d'aller casser les étalages de la grande surface, je passerais à la coopérative, à la chambre d'agriculture et à la caisse sociale faire le ménage ! 29 ».

Pour Nicoud, la relation identitaire à la violence entretenue par son mouvement ainsi que le maintien d'une très forte légitimité 30 dont ce dernier bénéficie sur une assez longue période au sein du groupe référent ${ }^{31}$, définissent une

24. Au sens de Wilson F., «Les groupes d'intérêt sous la Cinquième République. Test de trois modèles théoriques de l'interaction entre groupes et gouvernement ", Revue française de science politique, vol. 33, ${ }^{\circ} 2,1983$, pp. 220-254.

25. Voir $F F, 18$ avril 1969 , p. 3.

26. Extrait d'une allocution de fevrier 1955, cité in $F F, 7$ mars 1969.

27. Voir $F F, 28$ mars 1969, p. 6 ; FF du 26 sept 1969.

28. Voir en ce sens l'impact de "l'âge biologique » sur les mutations de carrière, relevé par Hugues E., op. cit., p. 176 et suiv.

29. Poujade P., L'Histoire sans masque, Cestas, Élytis éd. 2003, p. 191.

30. A l'opposé donc de la forclusion de la violence chez les paysans qui est largement tributaire du revirement de la perception sociale et médiatique des actions devenues contre-productives, voir Duclos N., Les violences paysannes sous la ve République, Paris, Economica, 1998. 
corrélation opposée à celle du poujadisme : le déclin du mouvement entraîne, à l'inverse, une réactivation de la politique du coup d'éclat savamment utilisée ponctuellement afin de remobiliser les troupes. En novembre 1971, le succès remporté lors des élections aux Chambres des métiers est directement imputable à l'incarcération de Nicoud. Son refus de faire appel de sa condamnation est alors uniquement motivé par la nécessité de relancer un mouvement dont les adhésions stagnent ${ }^{32}$. Dès lors, la désescalade se révèle beaucoup plus progressive. Même si l'ampleur et l'impact de la violence diminuent, la vie du CID-UNATI reste immanquablement ponctuée d'actions violentes (en 1976, $1980,1982, \ldots)^{33}$. Surtout, le leader du mouvement demeure le plus souvent acteur, si ce n'est l'instigateur, des actions violentes. En juillet 1976, il est inculpé pour incitation au meurtre après avoir déclaré à l'encontre des brigades spéciales de contrôle fiscal que « contre elles, la guerre est bien ouverte. Il faut qu'un de leurs membres perde la vie ou du moins soit pendu par les pieds 34 ». L'avocat du syndicat des impôts déplore cette dérive radicale qui contraint Nicoud « chaque fois qu'il prend la parole d'aller plus loin dans la violence » verbale 35 .

L'ampleur du mouvement de contestation ainsi fédéré et de l'investissement personnel contrevient dès lors pour les deux hommes, une fois le moment de lutte passé, à un retour à la vie commerçante ordinaire.

\section{Des attentes conjointes}

Réminiscences de la lutte. Le moment et le succès de la lutte ont radicalement transformé la vie des deux hommes. Même si, durant de nombreuses années, l'un et l'autre vont conserver leurs fonds de commerce, leur activité commerçante est devenue secondaire ${ }^{36}$. Leur carrière professionnelle épouse la carrière militante. Les deux leaders subissent tous deux les affres du reflux de leur statut et du rôle national d'interlocuteur tout puissant de l'État qu'ils ont pu respectivement acquérir et jouer. Le temps des salles bondées, des bras de fer avec les autorités publiques, des interviews et des rencontres avec les « grands » du pays, est bien révolu. Poujade et Nicoud aiment à rappeler que leur renommée au plus fort de la lutte a largement dépassé les frontières natio-

31. Voir pour une analyse des sondages, Mayer N., La boutique contre la gauche, Paris, Presses de Sciences Po, 1986 ; voir L'Objectif, n³ 30, mars-avril 1973.

32. Confirmé lors de l'entretien, 24 janvier 2008, un Restaurant, Neuilly sur Seine.

33. Gérard Nicoud comparaît le 29 décembre 1976 devant le TGI de Vesoul pour violences et voies de fait à agent de la force publique après une altercation lors d'une opération de collage d'affiches " antifisc ", cf. Le Monde, $1^{\mathrm{er}}$ janvier 1977, p. 8. Sur l'occupation d'un château, Le Monde, 18 novembre 1983, p. 12. Voir aussi « Gérard Nicoud incarcéré », Emission « Passez donc me voir », A2 - 28/02/1980, archives de l'INA, http://www.ina.fr.

34. Cité in Le Monde, 9 juin 1977, p. 18.

35. Ibid.

36. P. Poujade vend sa librairie en 1962, moment qui correspond aussi à la fin de l'aventure politique électorale nationale du poujadisme (même si des candidats se présentent encore sous l'étiquette poujadiste lors d'élections locales jusqu'en 1965). 
nales. Les multiples investissements professionnels successifs, faits de réussites et d'échecs, de revirements, se comprennent alors pour une grande part comme la manifestation d'une nouvelle quête tendant à la fois à revivre les heures glorieuses afin de " rester dans la lumière », mais aussi en tant que tentatives réitérées de se maintenir à la hauteur de leur histoire passée. En ce sens s’interprète le courrier envoyé par Gérard Nicoud après l'élection du président Nicolas Sarkozy de 2007 pour lui faire part de sa vision des transformations nécessaires du commerce et de l'artisanat : une volonté de poursuivre l'échange, d'égal à égal 37. Les soutiens électoraux publics apportés par Pierre Poujade jusqu'en 2001 (il appelle alors à voter pour Jean-Pierre Chevènement à la présidentielle de 2002) servent ainsi à (lui) rappeler que «Poujade » compte encore. Les propos tenus laissent à (faire) croire qu'il a même souvent contribué à «faire l'élection » 38 .

Un ensemble de points de convergence dans leurs trajectoires professionnelles et personnelles étaye cette pesanteur psychologique du moment de lutte, tel le maintien de manière cyclique de la pratique des «tours de France » qui avait pu contribuer à la fois à leur renommée parmi les artisans et les commerçants et à l'implantation de leur mouvement. Cette reprise de la route leur offre alors l'occasion de revivre l'émulation passée. Tous deux entretiennent une relation fusionnelle avec leur organisation qui les conduit également à multiplier les «vrais faux départs » (en 1978 pour Poujade; sur des périodes courtes successives pour Nicoud qui « abandonne » le poste de Secrétaire général en 1974, suite à son élection à la CANAM, puis le reprend en 1975, le quitte à nouveau brièvement en 1980) consécutifs aussi aux résultats, avortés, de leurs nouveaux investissements personnels, l'organisation faisant office de refuge. Mais les obstacles à l'acquisition de positions institutionnelles pour l'un, le passé de leader politique de l'autre, les inclinent surtout à multiplier les tentatives d'une voie de sortie «par le haut » d'un militantisme syndical devenu résiduel, et vis-à-vis d'une activité commerçante (trop) ordinaire, en cherchant tous deux à épouser la carrière politique.

Tentatives avortées de "reconversion » en politique. Le commun échec de ce réinvestissement en politique cache un rapport contrasté à l'arène électorale. La quête de mandat pour Poujade ne saurait définir une quelconque « reconversion » puisqu'elle s'inscrit dans les tentatives cycliques de relance du poujadisme politique (et électoral) originaire. Elle demeure tributaire dans un premier temps de la structure des opportunités politiques, l'avènement des élections européennes au suffrage universel direct offrant une double voie d'entrée fondée sur la nature du mode de scrutin retenu, à la proportionnelle, et pour une élection rendue favorable aux mouvements anti-système ${ }^{39}$. L'intériorisation des contraintes institutionnelles (l'échec de ses appels à la

37. Cette correspondance, selon G. Nicoud, ne reçoit pas de réponse. Cf. entretien cité.

38. «Le 10 mai 1981, écrit-il, une fois encore, les poujadistes ont pesé dans la balance électorale », cf. Poujade P., op. cit., 2003, p. 194. 
dissolution de l'Assemblée nationale en mars 1979 et au passage à un mode de scrutin à la Représentation proportionnelle pour les élections législatives) entraîne une conversion à la logique du régime de la ve République qui le mènera même, après son ralliement à François Mitterrand, à envisager à l'automne 1981 la création d'un nouveau mouvement, intégré, favorable au gouvernement 40 .

L'investissement de l'arène électorale lors des européennes de 1984 par Gérard Nicoud en tête de la liste « antipoliticienne » d'Union des travailleurs indépendants pour la liberté d'entreprendre (UTILE), liste socioprofessionnelle composée de seuls indépendants et patronnée par une alliance entre le CID-UNATI et l'UDCA ${ }^{41}$, intervient, à l'encontre des idéaux apolitiques initiaux, dans le cadre d'une conjoncture critique pour son mouvement. L'entérinement définitif du déclin de l'organisation 42 est consécutif, notamment, à la restructuration des organisations conventionnelles qui ont choisi de faire taire leurs divisions afin de contrer définitivement les protestataires 43 . Pourtant, cette voie de sortie par le politique se comprend moins comme l'expression d'une simple (re)conversion «stratégique » personnelle que comme la manifestation des dispositions antérieures, celles d'un fils de fonctionnaire de la municipalité marseillaise, communiste et militant de la CGT, et qui a connu une socialisation politique précoce ${ }^{44}$. Pour autant, chez lui, le désengagement militant qui suit sa candidature aux européennes (Nicoud quitte définitivement le CID-UNATI au Congrès de septembre 1984) n'est pas seulement consécutif de l'état de l'organisation et de l'échec de sa « reconversion » (la liste n'obtient que $0,68 \%$ des suffrages exprimés), mais également de l'effet de révélation et de réévaluation personnelle des coûts privés de la lutte 45 que cette impasse professionnelle a favorisé :

«Le jour où j'ai décidé de partir, en 1984, j’avais décidé que j'avais déjà perdu deux familles, deux divorces, que j’y avais laissé ma jeu-

39. Pierre Poujade crée l’Union pour la défense des libertés (UDL) afin de présenter une liste aux européennes de 1979, voir notamment Le Monde, du 23 septembre 1978, p. 10 ; 8 novembre 1978 , p. $10 ; 26$ avril 1979 , p. 42.

40. Le Monde, 3 décembre 1981, p. 8.

41. Poujade accepte d'apporter sa caution en figurant en fin de liste, cf. Le Monde, 5 avril 1984, p. 8 ; 12 juin 1984, p. $7 ; 25$ mai 1984, p. 10.

42. "Le déclin du cid-unati se confirme parmi les artisans », Le Monde, 24 nov. 1983, p. 36.

43. Cf. Milet M., « Parler d'une seule voix. La naissance de l'UPA et la (re)structuration du syndicalisme artisanal au tournant des années 70 », Revue française de science politique, vol. 58, $\mathrm{n}^{\circ} 3,2008$, pp. 483-510.

44. Cet engagement de « rupture apparente » avec l'apolitisme du leader syndicaliste se comprend comme l'envers de la reconversion dans l'humanitaire (« apolitique ») des médecins militants (gauchistes) politisés pour qui la reconversion n’est de même pas tant «stratégique » que tributaire d'une rupture bien antérieure au politique, cf. sur ces trajectoires Siméant J., «Un humanitaire "apolitique” ? Démarcations, socialisation au politique et espaces de réalisation de soi », dans Lagroye J. (ed.), La politisation, Paris, Belin, 2003, pp. 163-196.

45. Sur ce coût du privé, Gotteraux P., «Autodissolution d'un collectif politique. Autour de Socialisme et Barbarie », dans Fillieule O. (ed.), Le désengagement militant, Paris, Belin, 2005, pp. 85-86. 
nesse, une grande partie de ma santé ; (...) nous avions la loi Royer, nous avions eu le ministère qui est ce qu'il est malheureusement devenu, il y avait eu tout un tas de chose et j'estimais que j'arrivais au bout ; donc j'ai, et à une certaine limite de mon âge, et j'en ai marre, j'en ai marre parce que ça fait 17 ans et qu'est-ce qu'il me reste ? J'ai été plus loin que ce que j'avais promis...»

Cet extrait illustre combien indisponibilité biographique et contraintes de structure se confortent mutuellement dans la défection de l'action syndicaliste.

La différence du rapport à l'organisation-mère entre Nicoud et Poujade, puisque ce dernier conserve quant à lui la présidence de l'UDCA durant près de 30 ans (jusqu'en septembre 1983), se comprend alors dans le degré d'opportunité personnelle d'utilisation de la structure collective.

\section{Réinvestissements différenciés du capital militant : entre capital protestataire et capital violence}

La différence dans les modalités d'engagement de Pierre Poujade et de Gérard Nicoud pèse sur la teneur même du « capital militant » acquis dans la lutte, dès lors que l'on choisira de l'entendre à la fois comme acquisition d'un savoir-faire et d'une expérience 46 mais aussi, au travers du prisme interactionniste, en tant qu'instrumentalisation par ses titulaires de la perception des capacités qui leur sont prêtées par leur environnement et leurs interlocuteurs.

\section{Différenciations de "statuts»}

En effet, la commune acquisition d'un capital militant, fait notamment de l'apprentissage de la pratique des médias, d'une capacité de structuration de l'action collective, de repères sociaux et politiques, ne conduit pas chez les deux anciens leaders à un «statut » équivalent. Distinguant métier et carrière, Alexandre Pizzorno note ainsi que «tandis que la sécurité acquise par la mâ̂trise d'un métier est toujours dépendante d'une demande sur le marché, la garantie d'une position de carrière est due plutôt à une reconnaissance de statut social qui va bien au-delà de la garantie contractuelle 47 ».

Une carrière d'entrepreneur à facette multiple. Outre le fait qu'il ait acquis un sens aigu des déplacements sur des espaces sociaux variés, Poujade dispose d'une reconnaissance publique de ses aptitudes (en tant que fédérateur de projets, organisateur hors pair, tribun et négociateur) qui lui offre l'opportunité

46. En ce sens voir le dossier «Capital militant ", Actes de la Recherche en Sciences Sociales, $\mathrm{n}^{\circ} 155,2004$.

47. Pizzorno A., "Accumulation, loisirs et rapports de classe », Esprit, ${ }^{\circ}{ }^{2} 274$, juin 1959, p. $1000-$ 1016. 
de multiplier les investissements dans des sphères sociétales variées afin de prolonger son parcours professionnel ascendant en jouant d'une réelle multipositionnalité, mutuellement profitable. Car la voie politique n'est bien, pour lui, que l'une des possibilités offertes parmi d'autres.

Le déclin de l'action collective protestataire coïncide ainsi dès le début de l'année 1958 avec une réorientation constructive de la lutte menée par l'UDCA, effective à travers «l'Opération Confiance » qui vise à l'instauration d'un réseau de distribution en libre-service de gros pour les petits commerçants, susceptible d'abaisser leurs coûts et d'améliorer leur compétitivité. Romain Souillac note que, dans les premières années, le projet, loin de constituer un substitut à la lutte alors menée dans le champ politique, n'en est que son prolongement économique ${ }^{48}$. Toutefois, après le déclin du poujadisme politique, dès le début des années soixante, le groupement d'achat s'apparente bien à un resserrement, subi, de l'action sur le seul segment disponible : corporatif et socioprofessionnel. L'opération, en butte notamment à l'opposition des grossistes et aux inquiétudes, légitimes, des éventuels participants qui doivent à l'origine s'engager financièrement, connaît alors de multiples boutures : créé au Congrès de l'UDCA de novembre 1957, le projet rebaptisé «Opération Vérité » est relancé au Congrès d'octobre 1963 ; mais face à la faiblesse du nombre d'acheteurs « rares et insaisissables 49 » (une « bonne quarantaine » (sic) sur les 100000 escomptés), l'ambition initiale est revue à la baisse. Le projet d'envergure laisse alors la place à une simple négociation par secteurs, géographiquement circonscrite ${ }^{50}$, et qui se réduit bientôt à un groupement d'achats non plus de professionnels, mais de consommateurs militants, bénéficiaires de l'offre UDCA ${ }^{51}$. Le projet professionnel est relancé au début des années 1970 et bénéficie d'un soutien financier grâce au partenariat réalisé avec l'une des principales familles de la grande distribution, créateur de Promodes, qui consolide la création d'une Société Anonyme. Poujade revendique au plus fort de l'activité jusqu'à 24000 adhérents ${ }^{52}$, mais il est permis de douter de la véracité des chiffres avancés, d'autant que la Société ne tarde pas à péricliter, et fait faillite au milieu des années 1970.

Ce projet, originairement appuyé sur l'UDCA, marque l'ambivalence de l'investissement de la sphère économique pour Poujade : l'action collective est désormais étroitement corrélée à ses projets personnels. Car cette mutation est

48. Sur la première mouture du Projet Confiance, voir Souillac R., op. cit., pp. 306-310; Poujade P., 2003, op. cit., pp. 170-171; voir aussi dossier arch. nat, F/7/15619 (consultation sous dérogation).

49. "Le point sur l'opération vérité », $F F, \mathrm{n}^{\circ} 490,5$ février 1965, p. 10.

50. "A l'avant-garde de l'opération Carburant : la région parisienne ", $F F, \mathrm{n}^{\circ} 488,22$ janv. 1965, p. 10.

51. Il se restreint à l'obtention de rabais, principalement sur les carburants, et à l'achat « groupé » de voitures de tourisme. Deux structures cohabitent alors : le Groupement interprofessionnel de l'entreprise libre (GIPEL) pour les achats professionnels, et la Société Quercynoise de Publicité (SQP) pour les « achats d'utilisateur », toutes deux domiciliées à Limoges.

52. Sur cette alliance cf. Poujade P., op. cit., 2003, pp. 179-180. 
également perceptible dans l'évolution des festivités annuelles de l'UDCA qui se muent au milieu des années 1960 en une véritable foire commerciale et agricole sans le nom 53 : les jeux et les débats s'accompagnent de démonstrations de machines agricoles et de proposition de vente de produits, dont le Maerl, engrais biologique issu de la récolte des algues de mer et dont le principal promoteur au sein de l'UDCA est le propre fils aîné de Pierre Poujade, qui apparaît comme directement intéressé à son développement ${ }^{54}$.

En d'autres termes, à compter du milieu des années soixante, le mouvement syndical résiduel sert de structure d'accompagnement et de diffusion de l'investissement personnel du secteur marchand alors engagé par le leader de l'UDCA. Ainsi, au début des années soixante-dix, le Château du Gouzou, situé à Sainte Croix dans l'Aveyron, propriété conjointe de Fraternité française et de l'UDCA, acheté au plus fort de la réussite du mouvement et qui abrita un temps son école des cadres, est transformé en un Centre de formation professionnelle avicole qui entend former à l'apprentissage de la production des oies et des canards ${ }^{55}$. Cette mutation d'affectation des locaux suit en fait l'une des réorientations professionnelles de Pierre Poujade qui crée au même moment une «ferme pilote » orientée vers « l'aviculture de luxe » ayant des perspectives de « revenus très importants » dans l'approvisionnement des grands restaurants en foie gras et gibier. Le journal du mouvement Fraternité française se fait alors porteur d'un projet de regroupement de producteurs en une structure syndicale seule à même de faire bénéficier ses adhérents des aides publiques. Contrairement pourtant à la présentation initiale, la création de la ferme pilote n'est pas antérieure au projet de syndicat initié par Pierre Poujade, mais concomitante. En d'autres termes, c'est bien l'action collective qui est un préalable à la bonne réussite (économique) de l'entreprise familiale. Cette instrumentalisation d'une UDCA restreinte à quelques milliers de partisans inconditionnels transparaît dans l'autre grand projet poujadiste : la réalisation $\mathrm{du}$ «Club des vallées ».

Après avoir vendu sa librairie en 1962, Pierre Poujade a en effet acheté un peu plus d'une dizaine d'hectares de terres, en friche et difficilement accessibles, situées dans les Gorges de l'Aveyron. Le lieu sert bientôt de camp de vacances d'été des jeunes de l'UDCA, l'UDJF (Union de défense de la jeunesse française) qui travaille aussi à la restauration du vieux corps de bâtiment. En visionnaire, Pierre Poujade perçoit le développement du tourisme de masse et, dès 1965, envisage la création d'une société de vacanciers fondée sur des adhérents actionnaires qui auraient la jouissance du terrain, avant que les

53. "A La Bastide démonstration de matériel agricole », $F F, 13$ aout 1965, p. 5 ; de même, le lancement des «festivals entreprises libres », en juillet 1966, est un échec financier. L'objectif est aussi de faire rentrer des fonds pour l'UDCA.

54. Face aux attaques sur le vrai et le faux Maerl, Yves Poujade réplique en évoquant « le prix que nous faisons sur notre marchandise départ usine ", voir $F F, 15$ octobre 1965, p. 11.

55. La direction en est confiée à son fils. 
contraintes juridiques, et sans doute la faiblesse des demandes, ne l'incline à envisager un projet de plus grande envergure qui vise à la création d'un véritable complexe touristique (avec vente de terrains, construction de villas, création d'activités sportives, nautiques et de restauration $\left.{ }^{56}\right)$. Une telle réalisation nécessite un investissement financier colossal qui n'est envisageable qu'après le réaménagement profond du site, du fait, notamment, de la construction nécessaire d'une digue et de l'aménagement de la route d'accès principale. En août 1967, les « circonstances » font que les fêtes annuelles du mouvement ne peuvent se tenir sur « le terrain habituel de Cabanne », et dès lors les militants de l'UDCA travaillent au réaménagement complet du terrain en vue de la bonne tenue de « la Kermesse rurale » du mouvement, organisée le 13 août à Labastide Levèque. La mise en viabilité du site est réalisée en quelques semaines grâce à l'action conjointe des militants ${ }^{57}$. Afin de faire taire ses contempteurs qui l'accusent d'être passé du côté de l'ennemi (les grandes surfaces ${ }^{58}$ via l'opération Confiance) ou de la grande finance (le complexe touristique), Pierre Poujade fait de la défense « des petits contre les gros » la ligne de conduite de ses actions, s'affichant sous les traits renouvelés du « petit paysan de Labastide » 59 investi dans la « terre rouergate ». Il ressort en définitive l'établissement post-protestataire d'un véritable système militant néo-patrimonial dont le profit économique personnel, jamais nié, n’en est pas moins fortement minimisé 60 .

Cette réussite, Poujade la doit à son élévation au rang de véritable label. Le « label Poujade » le définit désormais en véritable « personne ressource » 61 qui lui permet, outre son investissement dans la sphère économique, de réaliser une double immixtion dans la sphère de la représentation. Il devient le porte-drapeau d'une multitude de regroupements collectifs (président des producteurs de porcs de la région du Rouergue en octobre 1970, depuis 1980 de la Fédération nationale des syndicats de producteurs de Topinambours, vice-président de la Confédération des syndicats de producteurs de plantes alcooligènes...). Nommé par François Mitterrand au Conseil Economique et social (1984-1999), Poujade fait également office d'homme de missions du pouvoir, du fait de son label international (en Amérique Latine, aux Antilles,

56. Est ouverte en 1968 l'Auberge du Lac, tenue par Madame Poujade, dont la publicité paraît de manière régulière dans les pages de Fraternité française.

57. "Le défi est relevé », $F F, 4$ août 1967, p. 1. Le barrage est consolidé, une route est ouverte à travers la colline de Lortal jusqu’à Mousset.

58. Il est noté que sa « réapparition » est due « au lancement de la société anonyme Confiance au capital de 990000 Fr. ", Pierre Poujade devient «PDG », "Coucou le revoilà ! Pierre Poujade est remis en piste ", L'Objectif, n`39, mars 1974, p. 3.

59. $F F, \mathrm{n}^{\circ} 618,5$ oct. 1967.

60. Évoquant « la vallée heureuse » le journal du mouvement note que «sans prétendre à la philanthropie Pierre Poujade veut donner à son œuvre un rôle humain ", $F F, 20$ oct. 1967, p. 5 ; s'agissant de la ferme pilote il est précisé que « ce n'est pas non plus une œuvre philanthropique car on ne voit pas pourquoi un homme perdrait son temps et son argent alors que de toute évidence il a suffisamment de soucis et de responsabilité avec ses propres problèmes ", $F F, 6$ février 1970, page centrale.

61. Cf. Offerlé M., Sociologie des groupes d'intérêts, Paris, Montchrestien, 1994. 
en Roumanie). Contrairement à Gérard Nicoud, il bénéficie de ce fait d'importants réseaux sociaux. Alors qu'il souhaite relancer le projet Confiance en sollicitant le Président Mitterrand, il lui propose de confirmer l'engagement pris par Georges Pompidou auprès de Michel Joubert, secrétaire général de la Présidence, et qui avait pu suivre antérieurement ses projets sous la présidence gaulliste. La parenté de «combat » et de génération avec François Mitterrand, l'ancien ministre de l'Intérieur de la IVe République, s'avère sans doute également un atout considérable, et sa présence lors de cérémonies officielles étonne alors, au milieu des années 1980, le cercle étroit du pouvoir socialiste ${ }^{62}$.

Le référent violent. Gérard Nicoud a, lui aussi, bénéficié d'un « capital scolaire de substitution 63 » par l'activisme syndical, mais sans doute plus encore dans le cadre de ses périodes d'incarcération qui font office selon ses propres mots, $d$ ' « universités pénitentiaires 64 », et ce notamment lors de son plus long séjour, au cours duquel il est nommé bibliothécaire de la prison de Bonneville, et qu'il met à profit pour lire de nombreux ouvrages. Mais fondamentalement le principal handicap de reconversion résulte du référent violent qui fonctionne désormais pour lui comme un véritablement enfermement qui contraint sa « carrière 65 » quelle que soit par ailleurs son évolution de métier.

Prisonnier du cadre violent, il subit un véritable marquage susceptible dès les années 1980 de restreindre ses capacités d'activité. Ses condamnations pèsent un temps sur son engagement en politique 66 et il manque d'être privé de ses droits syndicaux ${ }^{67}$. L'absence de processus de notabilisation ${ }^{68}$ qui aurait permis de lui offrir une voie de sortie honorable dans le cadre des fonctions de représentation au sein des instances consultatives représentatives semble en ce sens moins la résultante d'une distanciation personnelle apparemment volontaire à l'encontre $d u$ « syndicalisme de salon » («...qu'il me restait quoi ? à finir président de Chambre de commerce avec la médaille du mérite, j'avais dit ceci et je le maintiens je ne finirai pas dans la peau d'un vieux con » ${ }^{69}$ ) telle qu'elle lui apparaît encore plus de vingt ans plus tard, que la conséquence de la faiblesse d'une accumulation des ressources mobilisables et des contraintes de parcours d'activiste violent. Sa rupture avec le CIDUNATI révèle la maturité acquise par l'organisation si l'on songe que l'autonomie du groupe vis-à-vis du créateur définit aussi un processus de dépersonnalisation, l'un des critères qui fonde l'achèvement de toute institutionnalisa-

62. Relevé avec ironie par Pierre Poujade.

63. Voir le dossier «Capital militant», ARSS, n¹55, déc. 2004.

64. Entretien cité.

65. Au sens de Pizzorno.

66. Le risque $d^{\prime}$ ' inéligibilité » du fait de ses condamnations antérieures hypothèque un temps son engagement aux élections européennes, Le Monde, 22 février 1984, p. 8.

67. Cf. Le Monde, 5 avril 1980, p. 30 ; 9 avril 1980, p. 12 ; 14 avril 1980, p. 12 ; 6 juin 1980.

68. Elle se comprend en l'espèce comme la reconnaissance d'un statut social légitime assis sur l'obtention de poste de représentation au sein d'institutions reconnues locales ou nationales (tel le Conseil économique et social).

69. Entretien cité. 
tion d'un mouvement. Mais cette rupture lui ôte aussi un centre-refuge, et la différence de génération entre le jeune leader et ses interlocuteurs politiques pèse sur le maintien de réseaux sociaux en politique d'autant que la teneur des actions militantes radicales les rend très improbables.

Ce référent violent, du fait de l'incapacité même de Gérard Nicoud à s'en démarquer, ne fonctionne pas comme seule contrainte d'environnement. Sa trajectoire personnelle est émaillée, comme on l'a vu (supra note 33), d'actes de violence, dont les tout derniers, de surcroît, remontent à peine à quelques années : au début des années quatre-vingt-dix, l'une de ses tentatives de réinvestissement de l'espace public et médiatique intervient dans le cadre de l'apparition en France des émeutes urbaines. Après les violences au Val-Fourré à Mantes-la-Jolie, il se rend en effet sur place et médiatise un projet d'insertion en entreprise auprès des jeunes ${ }^{70}$; et dans ses nouvelles activités de conseil, entamées au tournant du siècle, il perpétue de même à de nombreuses reprises la pratique des coups de force.

Face à de sérieuses difficultés financières après sa rupture de 1984, il « survit » selon lui grâce à l'aide de quelques amis et tente alors de réutiliser son savoir-faire en conseillant les associations de commerçants, sans qu'il puisse arriver à transformer cette aide rémunérée en une activité professionnelle à temps plein. S'en suivent des années de retour à une activité commerçante ordinaire : grâce aux liens maintenus avec d'anciens militants, il « fait les marchés » en fruits et légumes dans la région parisienne de la fin des années 1980 jusqu'en 1992, moment où il monte un nouveau syndicat interprofessionnel, le Centre national des entreprises - CNDE -, qu'il dirige encore actuellement. Le référent violent fonctionne alors comme un outil de cohérence biographique qui apporte de la continuité dans une «carrière » professionnelle chaotique ${ }^{71}$. Sa trajectoire personnelle est réinterprétée à l'aune de ce cheminement qui le conduit ainsi à insister, dans ses livres et les entretiens accordés, sur sa propre aptitude à ne jamais se laisser faire. Elle l'incline à plus de soixante ans à se définir désormais lui-même, de manière très symptomatique, en «mois de prison cumulés ». En 2008, dans la courte notice de présentation pour l'une des conférences-débats qu'il anime, une place importante est faite à cet aspect de son parcours. L'intervenant est présenté en ces termes : «Engagé dans une contestation active qui vaudra à Gérard Nicoud d'être condamné à la prison ferme (un an et demi en cinq fois), il accumulera plus de cent procès 72 ».

70. «Val-Fourré : Gérard NICOUD, les jeunes et l'insertion », Actualités régionales Ile-deFrance, FR3 - 05/07/1991, archives de l'INA, http://www.ina.fr.

71. Sur la dimension compréhensive du concept de «carrière » qui «s'applique à des significations intimes, que chacun entretient précieusement et secrètement, image de soi et de sa propre identité ", cf. Goffman E., Asiles. Etude sur la condition sociale des malades mentaux, Paris, Minuit 1968, p. 179.

72. Notice d'inscription à la conférence débat du 4 juin 2008 au Restaurant Comme chez soi, Strasbourg (consultation internet de l'auteur) 
On concédera que la pérennité du référent violent ne s'inscrit aucunement dans une ligne de conduite déterminée et immuable, mais demeure éminemment tributaire de l'échec des bifurcations biographiques et de l'effet des séquences de vie. En d'autres termes, l'incapacité pour Gérard Nicoud à transformer le capital militant en activité politique a aussi contrevenu à une mutation, possible mais non probable, de son rapport à la violence. L'investissement dans une carrière politique eût pu conduire à une normalisation du comportement ou à une prolongation de la déviance qui aurait servi de critère de différenciation en politique.

La grande habileté de Gérard Nicoud tient alors dans sa capacité à ne pas seulement subir ce marquage mais à parvenir, aux termes de tentatives multiples, à le convertir, in fine, en «capital violence ». En d'autres termes, la ressource qu'il tire de son militantisme est directement corrélée à sa nature violente : le rejet qu'inspire son engagement radical passé devient un label identitaire puisque l'on se souvient de lui par le type d'actions entreprises, mais il lui sert aussi très concrètement de ressource mobilisable dans ses nouvelles activités professionnelles auprès de l'administration fiscale, ou par l'attirance et la légitimité acquise de celui qui va au bout de ses convictions.

\section{La construction de deux rôles inédits:}

le petit patron d'État et le médiateur fiscal

Aux termes de leur trajectoire personnelle, l'ambivalence de la carrière militante et professionnelle des deux leaders protestataires réside dans la continuité du lien très étroit entretenu avec l'État.

Le petit patron d'État. L'ensemble des investissements de la carrière de Pierre Poujade depuis le milieu des années 1960 ne prend sens en effet qu'à la lumière des éléments de concordance biographique : le ralliement au régime de la ve République et le partenariat engagé avec le pouvoir politique en place conditionnent la réalisation de l'ensemble des projets collectifs et personnels menés par Pierre Poujade.

Le premier rapprochement avec le pouvoir date en effet de l'été 1966, au cours duquel un ministre accepte de participer aux festivités de l'UDCA, qui précède la reconnaissance, en plusieurs étapes, du mouvement et l'édification d'un solide partenariat. Dès le Congrès de Lyon, en juin 1966, devant l'évolution manifeste de la position du pouvoir gaulliste à son égard, Pierre Poujade décide, non sans provoquer quelques oppositions au sein de son mouvement, de cesser « les attaques systématiques contre la politique du gouvernement et les hommes qui la font 73 ». Le soutien au régime est entériné au Congrès extraordinaire du 30 janvier 1967 (87 voix contre 5). Le processus de légitima- 
tion ${ }^{74}$ mutuellement profitable s'instaure alors : le soutien désormais accordé par l'UDCA aux candidats nantis de l'investiture « Ve République » permet la venue des parlementaires et d'un ministre au Congrès du mouvement ainsi que la création d'une association parlementaire UDCA qui aurait compté pas moins de 96 membres en 1967. La reconnaissance en représentativité se trouve définitivement entérinée par la réception de Pierre Poujade par le Premier ministre, Georges Pompidou, en juin 1968, puis à l'Elysée le 29 juin 1970. Ce ralliement offre l'occasion au pouvoir gaulliste de faire des membres de l'UDCA ses mici dominici au sein d'un segment socioprofessionnel alors faiblement unifié et en carence d'interlocuteurs. Une mission « d'information fiscale » est confiée au mouvement ainsi « accrédité par les pouvoirs publics » ${ }^{75}$. L'UDCA devient même en province le porte-parole de l'intérêt à la création de la TVA auprès des artisans et commerçants. Ce ralliement au régime est présenté a posteriori par Poujade sous les traits grandiloquents des « accords Poujade-Pompidou » qui vont être prolongés sous le gouvernement Couve de Murville, puis sous la présidence pompidolienne, et repris sous François Mitterrand.

Ce changement d'orientation de l'UDCA correspond étroitement à la réactivation des projets avortés du mouvement dans la sphère économique et conditionne la réussite des nouvelles entreprises personnelles élaborées par son leader. Dès janvier 1967, le journal du mouvement note ainsi qu' " il est évident qu'avec l'appui du gouvernement et la bienveillance de l'administration l'opération Confiance adaptée aux besoins et aux moyens du jour pourrait être réalisée si le personnel humain valable est disponible » 76. Alors qu'initialement, Pierre Poujade, dans ses nombreux récits de vie et ses articles, minimise tout apport et subvention de l'État, en réalité les montages financiers sont assurés grâce à ce nouveau partenariat. Comme il l'écrit en 2003, « le projet Confiance reprend bientôt à la base sous le nom Confiance - Angoulême, entièrement financé par l'État 77 ». Le projet touristique de « la vallée heureuse » n'est de même rendu possible, après bien des difficultés administratives liées au réaménagement du site, que par la toute nouvelle bienveillance des services de l'État ${ }^{78}$, si ce n'est les prêts consentis ${ }^{79}$. La création du Centre

74. Sur ce processus cf. Mény Y., «La légitimation des groupes d'intérêt par l'administration française ", Revue française d'administration publique, $\mathrm{n}^{\circ} 39,1986$, pp. 99-110.

75. FF, 21 juillet 1967 , p. 1 et 3 .

76. $F F, \mathrm{n}^{\circ} 13$ janvier 1967.

77. Cf. Poujade P., op. cit., 2003, p. 199.

78. «Or, dans le cadre de l'équipement national, l'idée initiale pouvait être retenue, puisque c'est très exactement le même but que poursuit à son échelon le secrétariat d'Etat au Tourisme »; il s'agit de s'y prendre « autrement, c'est-à-dire en faisant entrer le projet dans le cadre de la loi » dans «Le Club des vallées Nouvelle formule », FF, 1 er juillet 1966, p. 6. "Il a fallu aussi la compréhension et l'aide amicale de l'administration générale, des ponts et chaussées et de l'EDF en particulier », in $F F, 4$ août 1967, p. 3.

79. Il note ainsi « un projet semblable ne pouvant que trouver un écho favorable du côté des ministères intéressés peut escompter 50 \% environ de crédit, direct ou indirect de l'Etat », $F F$, 2 février 1968, p. 4-5. 
de formation avicole est également directement imputable à l'agrément reçu du ministère de l'Agriculture. Dans les années 1980, François Mitterrand prolonge « l'accord » et les multiples projets d'agro-énergies alors lancés par Poujade ${ }^{80}$ sont appuyés par les différents ministères concernés qui offrent aussi une caution dans les partenariats avec des grandes entreprises (telles Peugeot et Renault). Les soutiens apparemment contradictoires de Pierre Poujade aux candidats aux élections présidentielles prennent un nouveau sens : ils se conçoivent dès lors, dans la continuité, à la lumière de ces réinvestissements qui le portent toujours à s'engager en faveur du pouvoir en place ou à l'encontre du sortant qui a trahi sa confiance (tel le «vote contre » ̀̀ l'égard du président Valéry Giscard d'Estaing en 1981 qui vise aussi celui qui a rompu les « accords Pompidou-Poujade» en mettant notamment un terme $\mathrm{au}$ «volant de réserves de la Caisse des dépôts et Consignations pour le projet Confiance $81 »)$. Le slogan « sortons les sortants » s'était, il est vrai, dès février 1967, mué en « chassez les revenants 82 ».

Au final, la carrière professionnelle et militante de Pierre Poujade postérieure à la lutte correspond ainsi selon nous à la figure inédite du petit patron d'État. Elle s'inscrit au carrefour des deux types idéaux classiquement distingués au sein du grand patronat 83 : ce nouveau rôle emprunte en effet aux «patrons d'État » les connivences politico-administratives et la nature des affaires menées en lien avec la sphère étatique (en l'espèce non pas les marchés octroyés mais les subsides), et aux «patrons privés » les propriétés sociales d'une « petite bourgeoisie » de niveau d'étude modeste ${ }^{84}$.

Le médiateur fiscal. Comme on l'a vu, jusqu'au début des années 1990, Gérard Nicoud a rompu, pour sa part, avec le militantisme syndical, mais entretient toujours des liens étroits avec un solide réseau d'amitiés construit au temps de la lutte. La rencontre fortuite au cours d'un repas avec un entrepreneur industriel d'une moyenne PME du Val-d'Oise en indélicatesse avec l’URSSAF de Paris, lui permet alors de renouer avec le militantisme passé. La réorientation professionnelle correspond en fait aussi à un nouvel échec de reconversion en politique qui survient semble-t-il à la même période ${ }^{85}$.

80. Poujade, 2003 , pp. 247 et suiv. ; voir aussi «Un combattant du Topinambour », Le Monde, 30 janvier 1984, p. IX. Il est noté «M. Poujade ne tarit pas d'éloges sur la bonne volonté du gouvernement et sur l'aide que lui accorde l'AFME » (l'Agence Française pour la Maîtrise de l’Energie). Dès 1958, il s'intéresse au carburant de substitution, le projet Makhonine.

81. Cf. Poujade P., op. cit., 2003, pp. 179-180.

82. FF, 24 février 1967 , p. 1 ; le slogan vise les personnalités de gauche de la IVe République dont F. Mitterrand et G. Mollet.

83. Bourdieu P. ; (de) Saint-Martin M., «Le patronat », Actes de la Recherche en Sciences Sociales, n²0/21, mars-avril, 1978, pp. 3-82.

84. Pierre-Paul Zalio à partir de parcours professionnels de grands dirigeants a judicieusement noté la nature trop clivée de la dichotomie Grande entreprise/ PME, public/privé, marquée par la typologie de P. Bourdieu et M. de Saint-Martin, cf. Zalio P.-P., « Les entrepreneurs enquêtés par les récits de carrières : de l'étude des mondes patronaux à celle de la grammaire de l'activité entrepreneuriale », Sociétés contemporaines, n68, 2007, pp. 59-82. 
Gérard Nicoud accepte de faire office de négociateur informel. Il bénéficie alors de l'image du « coup de force » car la simple apposition de son nom sur un courrier au sein duquel il reprend « [son] ton syndicaliste » virulent suffit à lui faire obtenir un rendez-vous auprès du président de l'URSSAF de Paris. Il note avec amusement : " J'apprendrai de plus en plus au fur à mesure qu'en définitive on se souvient plus de moi du côté de l'administration que du côté des commerçants [rire] et que j'ai plus de félicitations du côté de l'administration que du côté des commerçants 86 ». Son passé resurgit sous la forme renouvelée mais persistante d'un capital violence :

«Je vais vous dire une chose : mon passé il est fantastique, parce que ce passé de violence, mais aussi pardonnez-moi de me flatter un peu, d'honnêteté, parce que le meilleur compliment c'est quand un directeur des impôts me reçoit, personnellement, il me traite pas d'égal à égal mais avec du respect il me dit vous n'avez pas changé : je lui réponds c'est un compliment, il me dit : oui c'est un compliment. Pourquoi ? Parce que je crie toujours, parce que je prends des risques, parce que ma dernière condamnation elle remonte à Bobigny il y a cinq-six ans quand je suis allé chercher manu militari les dossiers chez un expert-comptable indélicat pour sauver une entreprise de neuf employés, là j'ai pris deux mois de prison avec sursis et j'ai repris 5000 balles d'amendes, voilà où je n'ai pas changé ».

Cette première entrevue lui permet non seulement de régler l'affaire mais d'engager un processus de remobilisation collective :

«Et là-dessus ça démarre mal, y a le directeur et les deux directeurs adjoints, voyez y a le côté officiel, moi je suis avec mon ami [NB : un avocat, ancien du CID-UNATI] et on trouve une solution, et làdessus j'étais prêt à me lever parce que là moi je pense que j'avais fini, je retourne à mes fruits et légumes, et là y a dû avoir une confusion justement, CID-UNATI machin, - et pour vos autres adhérents que voulez-vous ? J'en ai pas d'adhérents. Et au lieu de dire, non mais attendez, et je dis, ben pour mes adhérents voilà ce que je souhaite et je mets, comme ça j'improvise, une forme d'accord, qui dure depuis 15 ans maintenant»

Le succès de cette première médiation lui apporte le soutien de l'entrepreneur qui l'aide alors matériellement et lui permet de proposer ses services aux PME de la région Île-de-France. Il règle ainsi les 17 premiers cas qui lui sont

85. Gérard Nicoud annonce son adhésion au CNI d'Yves Briant et son souhait de présenter une liste CNI dans les Bouches-du-Rhône aux élections régionales, et d'être candidat aux cantonales à Aix-en-Provence, voir Le Monde, 7 déc. 1991, p. 10.

86. Entretien cité. 
soumis et crée alors en 1992 le Centre National des Entreprises (CNDE). Tout comme Pierre Poujade, la reconversion professionnelle s'accompagne d'une élévation et d'un élargissement du champ d'actions, puisqu'il est passé d'une défense des " petits » indépendants (commerçants et artisans avec un faible nombre de salariés) à la préservation d'entrepreneurs de très petites entreprises (TPE) et des petites et moyennes entreprises (PME) ${ }^{87}$.

L'absence de réseaux sociaux en politique est donc palliée par la « relation de confiance » désormais établie avec l'administration fiscale. Avec une grande acuité, Gérard Nicoud perçoit la possibilité d'un nouveau créneau, celui d'un « syndicalisme » patronal réinventé. Loin de chercher à reprendre la lutte radicale et protestataire de sa jeunesse, dans l'impossibilité biographique de recomposer un syndicalisme revendicatif traditionnel qui serait en rupture marquée avec son parcours et ses idéaux indéniables, il (co)invente le rôle de médiateur fiscal, modèle de comportement attendu qui répond à un double besoin des entreprises, soucieuses de négocier des arrangements financiers avec l'URSSAF, mais aussi de l'administration qui obtient ce faisant une garantie de recouvrement de fonds et s'évite les procédures contentieuses. Gérard Nicoud transforme ainsi ses contraintes biographiques en atout : son absence de diplôme ne pourrait lui permettre de créer une agence de conseil en entreprise, opération qui serait de surcroît financièrement difficile et risquée. Son savoir-faire syndicaliste et ses connaissances juridiques acquises lui offrent alors la possibilité de transposer dans le syndicalisme patronal une activité classique du syndicalisme salarié. Il crée en effet le CNDE non pas en association loi de 1901 mais sous la loi de 1884, qui lui offre l'opportunité de représenter les adhérents de son «syndicat professionnel » devant les différents tribunaux (Tribunal des Affaires de Sécurité Sociale, Conseil de Prud'hommes, Tribunal de Commerce). Alors même que les juristes « professionnels » sont intéressés au contentieux, Gérard Nicoud privilégie l'arrangement amiable, de même que ses interventions préservent de lourds frais d'avocats. Ce syndicalisme de conseil vit alors sur les cotisations des adhérents et permet aussi d'afficher une continuité d'action dans la distance ainsi maintenue avec les syndicats traditionnels liés à l'État par leurs contraintes de financement ${ }^{88}$. La liberté fièrement revendiquée repose sur cette totale autonomie financière. Ce segment d'activité lui permet aussi de ne pas s'engager dans une quelconque représentativité, ce qui le préserve à la fois d'une quête de militants et de la présentation de candidats aux élections consulaires. Gérard Nicoud sillonne désormais la France en organisant des conférences-débats, au

87. Fort du lien établi du fait de son nouveau mariage, il crée aussi l'Association « France Maroc Entreprise » d'aide à la coopération et au développement d'entreprises françaises sur le marché marocain. Voir aussi en ce sens : «M. Nicoud propose d'aider les PMI-PME », Le Monde, 7 mai 1986, p. 31.

88. Voir sur ce point Andolfatto D., Labbé D., «Faut-il aider les syndicats français ? » Le Débat, $n^{\circ} 142,2006$, p.119-141 ; Milet M., «Dialoguer pour exister ? Le syndicalisme artisanal en quête de légitimité par le dialogue social (archives) ", Terrains E Travaux, n 14, 2008, pp. 6889. 
sein desquelles il prodigue ses conseils «face à l'administration » (sur les délais de paiement, les vices de forme, les types de requêtes). Pour autant, comme pour Pierre Poujade, le rapport à l'État n'en est pas moins extrêmement ténu. Outre que son activité résulte des liens entretenus avec les directions fiscales d'Île-de-France, Gérard Nicoud cherche à développer un centre de formation à la médiation et à l'activité syndicale, qui serait susceptible, à terme, de bénéficier des subventions étatiques.

L'affichage du combat d'une vie « au service des petits » permet aux deux leaders protestataires de reconstruire une cohérence biographique dans des parcours qui ont largement conduit à rompre avec les idéaux initiaux de la lutte, notamment par une élévation de la représentation auprès des PME/PMI. L'imprégnation du rapport à la violence, bien plus marquée chez Gérard Nicoud, a dès lors pesé beaucoup plus fortement sur son parcours professionnel que sur celui de Pierre Poujade. La comparaison des trajectoires professionnelles présente deux modalités d'instrumentalisation du label protestataire, dans le cadre du maintien d'un support organisationnel ou d'une réinvention de l'activité syndicale. En ce sens, on voit aussi l'évolution et le recentrage des territoires géographiques et «imaginés » d'activité des deux anciens leaders : le premier, Pierre Poujade, s'est recréé un espace rural d'influence (la vallée du Mousset), là ou le second, Gérard Nicoud, s'appuie sur un territoire urbain et un segment d'activité restreint mais capté (le conseil fiscaliste en Îlede-France), une différenciation qui suit en cela la logique distincte d'implantation originaire du poujadisme et du nicoudisme. Tous deux ont poursuivi leur rapport ambigu avec l'État, dont on ne rappellera jamais assez qu'il ne constitue pas une rupture avec l'engagement passé, tant il est vrai que les deux frondes des petits définissent, dès l'origine, un appel à l'État plutôt que sa seule contestation. Car les mouvements sont avant tout marqués, au-delà des discours anti étatistes et fiscalistes, par des demandes de régulation d'un secteur d'activité. Au milieu des années cinquante, la fronde poujadiste est tributaire de l'arrêt de l'inflation et d'une politique fiscale rigoureuse imposée par Antoine Pinay, qui interviennent dans le cadre d'une recomposition endogène du secteur du commerce et des activités de proximité engagée dans le processus de modernisation. Le nicoudisme, pour sa part, s'oppose avant tout aux organisations professionnelles traditionnelles accusées de n'avoir pas su mieux prévoir les effets de l'entrée en vigueur de la loi relative au régime d'assurance vieillesse. Et de fait, les actions violentes vont contribuer à une réorientation du traitement étatique du secteur : réforme de l'assurance maladie des indépendants en 1970, première création en 1972 d'un ministère ad hoc du Commerce et de l'artisanat, loi Royer d'orientation du commerce et de l'artisanat en 1973. En définitive, l'un des célèbres aphorismes prononcés par Sacha Guitry à l'égard des femmes semble bien pouvoir être repris, à propos, pour nos deux leaders : «Je suis contre l'État... tout contre». 